\title{
Expected performance and simulated observations of the instrument HARMONI at the European Extremely Large Telescope (E-ELT)
}

Arribas, Santiago, Thatte, Niranjan, Tecza, Mathias, Goodsall, Timothy, Clarke, Fraser, et al.

Santiago Arribas, Niranjan A. Thatte, Mathias Tecza, Timothy Goodsall, Fraser Clarke, Roger L. Davies, Roland Bacon, Luis Colina, David Lunney, Evencio Mediavilla, Alban Remillieux, Dimitra Rigopoulou, Mark Swinbank, Aprajita Verma, "Expected performance and simulated observations of the instrument HARMONI at the European Extremely Large Telescope (E-ELT)," Proc. SPIE 7735, Ground-based and Airborne Instrumentation for Astronomy III, $77355 \mathrm{H}$ (6 August 2010); doi: 10.1117/12.857548

EviE Event: SPIE Astronomical Telescopes + Instrumentation, 2010, San Diego, California, United States 


\title{
Expected performance and simulated observations of the instrument HARMONI at the European Extremely Large Telescope (E-ELT)
}

\author{
Santiago Arribas ${ }^{\mathrm{a}}$, Niranjan Thatte ${ }^{\mathrm{b}}$, Matthias Tecza ${ }^{\mathrm{b}}$, Timothy Goodsall ${ }^{\mathrm{b}}$ Fraser Clarke ${ }^{\mathrm{b}}$, Roger L. \\ Davies $^{\mathrm{b}}$, Roland Bacon ${ }^{\mathrm{c}}$, Luis Colina ${ }^{\mathrm{a}}$, David Lunney ${ }^{\mathrm{d}}$, Evencio Mediavilla ${ }^{\mathrm{e}}$, Alban Remillieux ${ }^{\mathrm{c}}$, \\ Dimitra Rigopoulou ${ }^{\mathrm{b}}$, Mark Swinbank ${ }^{\mathrm{f}}$, Aprajita Verma ${ }^{\mathrm{b}}$
}

${ }^{\mathrm{a} C S I C}$, Instituto de Estructura de la Materia, Serrano 121, 28006-Madrid, Spain; ${ }^{\mathrm{b}}$ University of Oxford; ${ }^{\mathrm{C}} \mathrm{CRAL} ;{ }^{\mathrm{d} U K A T C} ;{ }^{\mathrm{e}} \mathrm{IAC} ;{ }^{\mathrm{f}}$ University of Durham

\begin{abstract}
HARMONI has been conceived as a workhorse visible and near-infrared (0.47-2.45 microns) integral field spectrograph for the European Extremely Large Telescope (E-ELT). It provides both seeing and diffraction limited observations at several spectral resolutions $(\mathrm{R}=4000,10000,20000)$. HARMONI can operate with almost any flavor of AO (e.g. GLAO, LTAO, SCAO), and it is equipped with four spaxel scales $(4,10,20$ and 40 mas) thanks to which it can be optimally configured for a wide variety of science programs, from ultra-sensitive observations of point sources to highangular resolution spatially resolved studies of extended objects. In this paper we describe the expected performance of the instrument as well as its scientific potential. We show some simulated observations for a selected science program, and compare HARMONI with other ground and space based facilities, like VLT, ALMA, and JWST, commenting on their synergies and complementarities.
\end{abstract}

Keywords: Infrared instruments, visible instruments, Integral Field Spectrographs, ELT

\section{INTRODUCTION: THE INSTRUMENT}

HARMONI is one of the phase-A European Extremely Large Telescope (E-ELT) instrument studies funded by ESO ${ }^{1}$. It is a visible and near-infrared $(0.47$ to $2.45 \mu \mathrm{m})$ integral field spectrograph, providing the E-ELT's core spectroscopic capability, over a range of resolving powers from $\mathrm{R}(\equiv \lambda / \Delta \lambda) \sim 4000$ to $\mathrm{R} \sim 20000$. The instrument generates simultaneous spectra of $\sim 32000$ (8000) spatial pixels in the near-infrared (visible) arranged in a 2:1 aspect ratio contiguous field. This FoV shape is ideally suited for nodding-on-IFU, allowing a high quality sky subtraction.

HARMONI has a range of spatial pixel (i.e. spaxel) scales, which permit the user to optimally configure the instrument for a wide range of science programs. The coarsest scale (with spaxels of 0.04 ") provides a 5" x 10" FoV, well suited to seeing limited observations, and the finest scale a factor of 10 finer (4 milli-arcseconds / spaxel), Nyquist sampling the diffraction limit of the E-ELT at near-infrared wavelengths. With its large range of spaxel scales, HARMONI can easily adapt to any flavour of adaptive optics -- indeed it is designed to work with GLAO, LTAO and SCAO, or even with no $\mathrm{AO}$ at all. HARMONI is conceived as a workhorse instrument, addressing many of the key E-ELT science cases.

It has been designed to exploit the E-ELT's scientific niche in its early years, starting at first light. At close-to-diffraction limited scales, it will capitalize on the $\mathrm{D}^{4}$ sensitivity gains of the E-ELT, providing unprecedented sensitivity and spatial resolution, which when put together, will transform the landscape in observational visible and near-infrared astronomy. Even in seeing limited conditions (or when AO cannot produce high Strehl ratios), HARMONI provides impressive gains with respect to the current generation of VLT instruments, e.g. a gain of $\sim 25$ in speed relative to MUSE at the ESO-VLT. As we will comment in Section 3, HARMONI has high complementarity and synergy with ALMA and JWST. Further details about the instrument can be found in these proceedings ${ }^{1}$.

Ground-based and Airborne Instrumentation for Astronomy III, edited by lan S. McLean,

Suzanne K. Ramsay, Hideki Takami, Proc. of SPIE Vol. 7735, 77355H · @ 2010

SPIE · CCC code: $0277-786 X / 10 / \$ 18 \cdot$ doi: $10.1117 / 12.857548$

Proc. of SPIE Vol. $773577355 \mathrm{H}-1$ 


\section{SCIENCE POTENTIAL}

The E-ELT will revolutionize astrophysics when it comes into operation. After centuries of telescope construction designed largely to provide for greater collecting area, E-ELT is designed as an adaptive telescope with dramatic gains in both sensitivity and spatial resolution. E-ELT will be commissioned after ALMA has been operational for a few years and after the launch of JWST. In this section we show that HARMONI, a visible/near-infrared integral field spectrograph, is ideally suited to exploit the unique advantages of E-ELT. The science cases for HARMONI address many of the major questions of astrophysics, the study of distant supernovae as the key diagnostics of dark energy, the nature of other planetary systems, the role of black holes and AGN in limiting the growth of the most massive galaxies, the properties of the highest redshift objects and the epoch and mechanism for re-ionisation.

\subsection{Sensitivity}

The expected point and extended source sensitivities of HARMONI under LTAO operations (see Table 1) have been computed using the E-ELT spectroscopic ETC, with some additional information on expected LTAO performance provided by the ATLAS consortium ${ }^{2}$. Instead of presenting a complete table of sensitivities for all wavelength ranges and spectral resolutions, we chose two atmospheric bands, $\mathrm{R}$ and $\mathrm{H}$. These are representative of the visible and nearinfrared sensitivity of HARMONI, and provide a full overview of its expected performance, without providing unnecessary details. $\mathrm{AB}$ magnitudes are used throughout.

The following assumptions underlie the computations of limiting magnitudes:

- Signal-to-noise of 5 per spectral pixel for point sources (averaged over all pixels lying between bright $\mathrm{OH}$ night sky lines) or per spectral and spatial pixel (extended source) in 5 hours.

- $\quad 0.8$ " atmospheric seeing, airmass = 1.0, LTAO correction, $900 \mathrm{sec}$ individual exposures, RON of 8e- (near-IR), 2e- visible.

Table 1- Point and extended source sensitivity of HARMONI in the R and H bands under LTAO operations

\begin{tabular}{|r|r|r|r|r|r|r|r|r|r|}
\hline Spectral & \multicolumn{2}{|c|}{4 mas } & \multicolumn{2}{c|}{10 mas } & \multicolumn{2}{c|}{20 mas } & \multicolumn{2}{c|}{40 mas } \\
\hline Resolution & \multicolumn{1}{|c|}{$\mathrm{R}_{\mathrm{AB}}$} & \multicolumn{1}{c|}{$\mathrm{H}_{\mathrm{AB}}$} & \multicolumn{1}{c|}{$\mathrm{R}_{\mathrm{AB}}$} & \multicolumn{1}{c|}{$\mathrm{H}_{\mathrm{AB}}$} & \multicolumn{1}{c|}{$\mathrm{R}_{\mathrm{AB}}$} & \multicolumn{1}{c|}{$\mathrm{H}_{\mathrm{AB}}$} & $\mathrm{R}_{\mathrm{AB}}$ & \multicolumn{1}{c|}{$\mathrm{H}_{\mathrm{AB}}$} \\
\hline & \multicolumn{8}{|c|}{ Point source (mag) } \\
\hline 4000 & 24.2 & 26.6 & 25.3 & 27.4 & 25.3 & 27.4 & 25.4 & 27.0 \\
\hline 10000 & 23.2 & 25.6 & 24.4 & 27.1 & 25.0 & 26.7 & 25.0 & 26.4 \\
\hline 20000 & 22.5 & 25.4 & 23.5 & 26.6 & 24.1 & 26.5 & 24.4 & 26.1 \\
\hline & \multicolumn{8}{|c|}{ Extended source (mag/ sq. arcsec) } \\
\hline 4000 & 19.2 & 18.2 & 21.0 & 19.3 & 21.9 & 20.3 & 22.7 & 21.1 \\
\hline 10000 & 18.2 & 17.8 & 20.1 & 18.9 & 21.5 & 19.8 & 22.3 & 20.6 \\
\hline 20000 & 17.5 & 16.7 & 19.5 & 18.5 & 21.0 & 19.4 & 22.2 & 20.3 \\
\hline
\end{tabular}

It can be seen that at near-infrared wavelengths, the best sensitivity for point sources is at the 10 and 20 mas spaxel scales. Starting with 40 mas spaxels, as one moves to finer spaxel scales, the sky background contribution gets smaller, but, at least initially, the source flux does not decrease. However, detector noise starts to play a bigger role. Once the telescope PSF is resolved (4 mas spaxels), the source flux per spaxel drops too, and sensitivity is lowest. However, for extended sources, the largest spaxels give the highest sensitivity, as would be expected. Although 10 and 20 mas spaxel scales have very similar sensitivities, two scales are provided so as to best match the sampling and the FoV to the properties of the source being studied. In Tables 2 we present similar tables under GLAO operations. 
Table 2- Point and extended source sensitivity of HARMONI in the R and H bands under GLAO operations

\begin{tabular}{|l|c|c|c|c|c|c|c|c|}
\hline Spectral & \multicolumn{2}{|c|}{4 mas } & \multicolumn{2}{c|}{10 mas } & \multicolumn{2}{c|}{20 mas } & \multicolumn{2}{c|}{40 mas } \\
\hline Resolution & $\mathrm{R}_{A B}$ & $\mathrm{H}_{\mathrm{AB}}$ & $\mathrm{R}_{\mathrm{AB}}$ & $\mathrm{H}_{\mathrm{AB}}$ & $\mathrm{R}_{\mathrm{AB}}$ & $\mathrm{H}_{\mathrm{AB}}$ & $\mathrm{R}_{\mathrm{AB}}$ & $\mathrm{H}_{\mathrm{AB}}$ \\
\hline & \multicolumn{7}{|c|}{ Point source (mag) } \\
\hline 4000 & 20.4 & 20.8 & 22.0 & 21.9 & 22.9 & 22.7 & 23.7 & 23.4 \\
\hline 10000 & 19.4 & 20.2 & 21.3 & 21.4 & 22.4 & 22.2 & 23.2 & 23.0 \\
\hline 20000 & 18.7 & 19.7 & 20.7 & 21.0 & 21.9 & 21.8 & 22.8 & 22.5 \\
\hline \multicolumn{8}{|c|}{ Extended source (mag/sq. arcsec) } \\
\hline 4000 & 19.2 & 18.4 & 20.8 & 19.5 & 21.7 & 20.3 & 22.5 & 21.1 \\
\hline 10000 & 18.3 & 17.8 & 20.1 & 19.0 & 21.1 & 19.8 & 22.0 & 20.6 \\
\hline 20000 & 17.6 & 17.3 & 19.4 & 18.6 & 20.7 & 19.4 & 21.6 & 20.2 \\
\hline
\end{tabular}

\subsection{HARMONI: A workhorse instrument}

We have looked at the HARMONI instrument parameters in light of the science now believed to be carried out at the EELT, represented by the Design Reference Mission (DRM) suite of programs. Table 2 lists the key instrument criteria, and their compliance with the various DRM science cases. A tick indicates that the relevant parameter is fully sufficient to carry out the science, a dash that it is adequate but not optimal, and a cross that it is unsatisfactory. Note that multiplex factor is not included, as it is not an instrument parameter.

The table highlights the fact that HARMONI is a workhorse instrument for the E-ELT, designed to carry out a wide variety of scientific observing programs. It is clear that HARMONI is the optimal choice for several of the science cases of the DRM, and more than adequately carries out the majority of the DRM science cases. It can contribute to the studies of the Initial Mass Function (IMF) and Young Star Clusters (i.e. S5) in a follow-up fashion, where spectroscopy is required. The green colour indicates those cases where the parameters of HARMONI are a good match.

Table 3- Compliance of the HARMONI instrument parameters in achieving the science goals of the various ELT / DRM science cases

\begin{tabular}{|l|c|c|c|c|c|}
\hline \multicolumn{1}{|c|}{ DRM case } & FoV & $\begin{array}{c}\text { Angular } \\
\text { Resolution }\end{array}$ & $\begin{array}{c}\text { Spectral } \\
\text { resolution }\end{array}$ & $\begin{array}{c}\text { Wavelength } \\
\text { range }\end{array}$ & Sensitivity \\
\hline S3 - exoplanets & $\checkmark$ & $\checkmark$ & $\checkmark$ & $\checkmark$ & $\checkmark$ \\
\hline S9 - circumstellar disks & $\checkmark$ & $\checkmark$ & - & $\checkmark$ & $\checkmark$ \\
\hline S5 - IMF and Young Star Clusters & $\mathbf{x}$ & $\checkmark$ & $\mathbf{x}$ & $\checkmark$ & $\checkmark$ \\
\hline G4 - resolved stellar pops & - & $\checkmark$ & $\checkmark$ & $\checkmark$ & $\checkmark$ \\
\hline G9 - black holes and AGN & $\checkmark$ & $\checkmark$ & $\checkmark$ & $\checkmark$ & $\checkmark$ \\
\hline C10 - physics of high z gals & - & $\checkmark$ & $\checkmark$ & $\checkmark$ & $\checkmark$ \\
\hline C4 - first light & $\checkmark$ & $\checkmark$ & $\checkmark$ & $\checkmark$ & $\checkmark$ \\
\hline C7 - enrichment of IGM & $\checkmark$ & $\checkmark$ & $\mathbf{x}$ & $\mathbf{x}$ & $\checkmark$ \\
\hline C2 - expansion history of Universe & $\checkmark$ & $\checkmark$ & $\mathbf{x}$ & $\mathbf{x}$ & $\checkmark$ \\
\hline
\end{tabular}

\subsection{Simulated Observations: Physics of high redshift ULIRGs}

As an illustrative example, here we present in more detail the study of high-z Ultraluminous Infrared Galaxies (ULIRGs) with HARMONI. This program is part of theme C10: the physics of high-z galaxies.

Large area multi-wavelength galaxy surveys spanning X-ray to radio wavelengths to great depths have revealed much about the global properties of high redshift galaxies such as their luminosity functions, stellar and total masses, sizes, spectral energy distributions as well as their evolution with redshift. These studies have helped us to develop and constrain the theory of galaxy assembly, however, we have little or no data to test the physical mechanisms at work, as probing the internal structure of high redshift galaxies is largely beyond our current capabilities. Detailed studies with HARMONI of the internal kinematics, spatially resolved stellar populations, dust distribution, ionization structure, nuclear properties, and interaction with the IGM will show how the different physical processes involved are interrelated, 
and how they give rise to the integrated physical properties we observe. Here we consider how HARMONI will be used to study the formation of the most massive galaxies and the processes at work shaping ordinary galaxies.

Ultra-luminous and luminous infrared galaxies, U/LIRGS, are rare in the local Universe but are much more common at high redshift. They are the likely progenitors of some of the most massive galaxies. U/LIRGs have large amounts of gas and dust, and are undergoing an intense star formation in their (circum)nuclear regions. This starburst activity is believed to be their major energy reservoir, although AGN activity may also be present. In many cases ULIRGs show clear signs of an on-going merging process, thanks to which the interstellar media experiences loss of angular momentum and the gas is transported inward. Close to the centre the gas is concentrated and compressed producing an intense star formation on scales of less than a few kiloparsecs. Low angular momentum gas produced in part by stellar mass loss in the starburst zone may fall into the nucleus proper and accrete onto the central massive black hole. The presence of an AGN and the associated energetic outflows may provide the feedback that limits the growth of massive galaxies. We can use HARMONI to test all these processes and investigate if U/LIRGS are local examples of the formation of the most massive galaxies at $\mathrm{z} \sim 2$ by utilising both the 10 mas $(\sim 100 \mathrm{pc})$ and $40 \mathrm{mas}(\sim 400 \mathrm{pc})$ scales. In particular we can probe the circumnuclear environment to detect nuclear disks or rings, non-rotational flows such as starburst-induced superwinds, tidally induced motions, or nuclear gas inflows. We will be able to establish the dynamical mass of the host galaxies using ionised gas rotation curves (and independently from cold molecular gas using ALMA). Extranuclear shocks can be identified from their LINER-like spectrum, and high velocity dispersion. Figure 1 shows how HARMONI will resolve the detail in the data cubes of high redshift galaxies. Structures at scales of up to 100pc will be resolved (and studied spectroscopically). In the example shown in Figure 1 the knots of star formation along the ring are clearly seen at $\mathrm{z} \sim 2$. Note that this redshift is about the most unfavorable in terms of the linear scale / arcsec ratio.
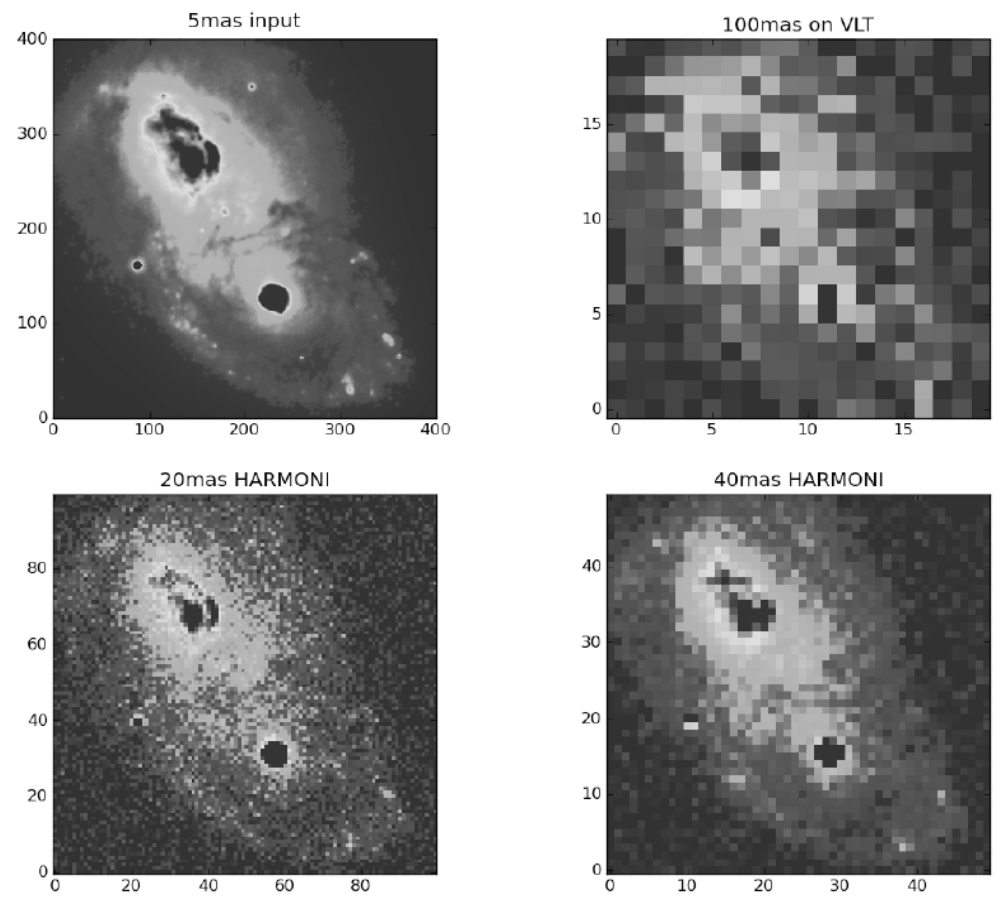

Figure 1: Simulations showing an ULIRG at $\mathrm{z} \sim 2$, imaged in the $\mathrm{K}$ band (rest frame H $\alpha$ ), using HARMONI at different spaxel scales (20 and 40 mas, bottom panels). The corresponding VLT view is shown at top-right, and the simulation input at top-left. SCAO/LTAO performance is assumed.

In general, the combination of high spatial and spectral resolution provided by HARMONI + LTAO will allow the processes occurring within high-z galaxies to be probed on scales of individual HII regions. Before LTAO is available it is possible that significant progress can be made with SCAO correction. Although today the surface density of spectroscopically confirmed $\mathrm{z} \sim 2$ galaxies which lie within $\sim 30$ " of a $\mathrm{R}<16$ mag star is limited, wide area narrow-band imaging surveys promise to yield significant numbers of high-z galaxies close enough to bright stars to provide high 
Strehl. The recent High-z Emission Line Survey (HiZELS) ${ }^{3}$, if extrapolated by a factor 10 in flux ${ }^{4}$, should yield $\approx 500$ galaxies across 5 sq-degrees $(\sim 150$ at each epoch of $z=0.84, z=1.47$ and $z=2.23)$ which lie within 20 " of $\mathrm{R}<15.0$ stars and have $\mathrm{H} \alpha$ fluxes brighter than $10^{-17} \mathrm{ergs} / \mathrm{s} / \mathrm{cm} 2 / \AA$. This provides a large, flux-limited sample of identically selected $\mathrm{H} \alpha$ emitters at epochs spanning the peak of the cosmic SFR distribution, and a sample of relatively low-luminosity galaxies (with SFR down to $<1 \mathrm{M} \odot / \mathrm{yr}$ ) which are comparable to the Milky Way at $\mathrm{z}=0$, but seen at $\mathrm{z} \sim 1-2$. In the era of VISTA, many tens of square degrees will have been surveyed, proving no shortage of targets for this program.

\section{COMPARISON WITH OTHER GROUND AND SPACE FACILITIES}

\subsection{Comparison with VLT}

HARMONI provides impressive gains with respect to the current generation of VLT instruments. For instance at visible wavelengths it will have a gain of $\sim 25$ in speed relative to MUSE at the ESO-VLT. At diffraction limited near-infrared observations HARMONI takes full advantage of the $\mathrm{D}^{4}$ gain factor.

\section{2 Synergies with ALMA}

HARMONI will have similar angular resolution to ALMA. Together they will elucidate the evolutionary connections between gas and dust, planets and star formation, AGNs and galaxy assembly. By probing metallicities of main sequence stars deep into the Galactic halo, HARMONI will augment the picture of halo assembly built by GAIA. The combination of ALMA and the E-ELT is ideally suited to the study of early galaxy evolution from high redshifts to today. The interplay of these giant facilities can address many questions, such as: i) the fraction of star formation due to major vs. minor mergers vs. steady accretion?; ii) how do discs evolve and how do bulges form?; iii) what drives the internal evolution of high-z star-forming galaxies?; iv) how does chemical enrichment proceed?; v) how does the co-evolution of black holes, AGN and their host galaxies vary with redshift?

\section{3 Complementarity with the Integral Field Unit of NIRSPec / JWST}

The JWST is roughly seven times smaller in radius (over 40 times in area) than E-ELT, but because it will operate at L2 has an extremely low infrared background. Comparing the capability of HARMONI to the IFU of NIRSpec is not straightforward due to their different basic parameters as well as programmatic issues. While the IFU of NIRSPec will arrive first providing an angular sampling of 100 mas, HARMONI will subsequently provide spatial resolved spectra, at resolutions ranging from 100 mas down to 8 mas (E-ELT diffraction limit). In terms of spectral resolution the IFU of NIRSpec takes full advantage of its low background environment working efficiently at low-median resolutions (100, $1000,2700)$. On the other hand HARMONI provides high spectral resolution $(4000,10000,20000)$, minimizing the effects produced by the background.

The sensitivities achieved for point sources by the IFU of NIRspec on JWST (R2700) and HARMONI (R4000) are compared in Figure 2 using several cases (LTAO with 20mas spaxels and GLAO and seeing-limited with 40mas spaxels). For HARMONI the exposure times were estimated with the E-ELT Spectroscopic ETC (v 2.14) and using the sensitivity tables and curves for NIRSpec. Four wavelengths were selected within the J, H, K and L atmospheric transmission bands in regions free of telluric emission lines according to the ETC $(1.255,1.600,2.240,3.52$ microns). The latter is not covered by HARMONI, but it is included to describe better the general behavior. For wavelengths different from the ones selected, HARMONI may have a much worse sensitivity as it can be inferred from the atmosphere emission and transmission curves presented in the lower panels. Leaving details aside, the figure illustrate two main points: i) HARMONI has a very competitive sensitivity at all spatial resolutions at wavelengths up to 2.5 microns (if free of telluric lines and within the transmission band), whereas the IFU of NIRSpec is unbeatable for longer (thermal-IR) wavelengths, and ii) LTAO should produce an important gain in sensitivity with respect to GLAO and seeing limited observations.

It is important to note that NIRSpec also has $\mathrm{R}=100$ and $\mathrm{R}=1000$ spectroscopic options, which provide a much higher sensitivity (especially for continuum work), and a multi-object mode, which provides a multiplexing gain larger than 
100 , in all cases with a pixel scale of 100 mas. On the other hand, in addition to $\mathrm{R}=4000$, HARMONI has $\mathrm{R}=10000$ and 20000 modes with a variety of spaxel scales. In summary, HARMONI and the IFU of NIRSpec occupy different regions in the instrument parameter space, providing outstanding complementarity for future spatially resolved spectroscopic studies at optical and near infrared wavelengths.

HARMONI also offer excellent synergy and complementarity with other NIRSpec modes, and other JWST instruments. It is easy to imagine HARMONI follow up observations of objects first discovered by NIRCAM, NIRSpec/MOS, MIRI and TFI. Therefore, HARMONI will drive transformational science showing an excellent complementarity with other major facilities like ALMA and JWST.

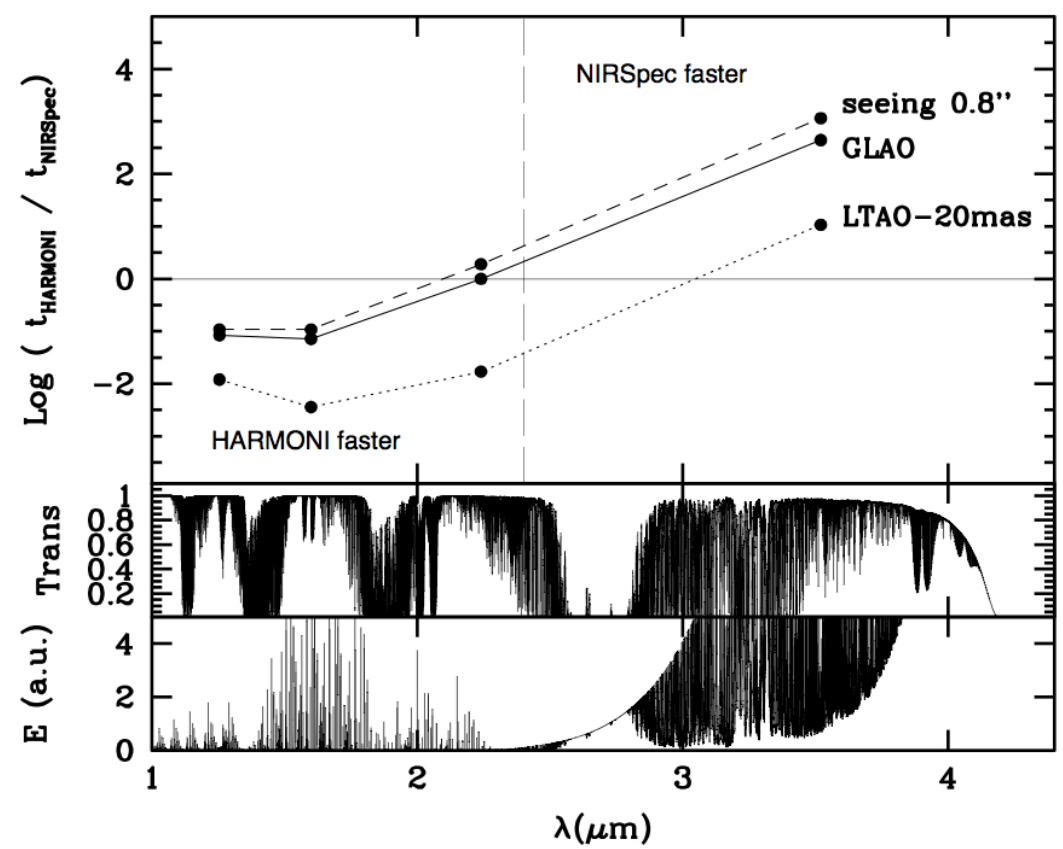

Figure 2. Speed comparison of HARMONI / ELT (at R=4000) and the integral field unit of NIRSpec / JWST (at R=2700). The results include HARMONI seeing limited (40mas spaxel scale), GLAO (40mas), and LTAO (20mas) observations of point sources. Computations have been done at four wavelengths selected to be in a clean region, between sky emission lines $(1.255,1.600,2.240$, and 3.520 microns, the latter outside the HARMONI spectral range). The points are connected with lines for clarity reasons. However, results for wavelengths between those selected may vary substantially. On one hand regions between the bands are severely absorbed by the Earth's atmosphere (see atmospheric transmission profile presented in the intermediate panel). On the other hand the presence of strong emission lines within the band (see the lower panel) reduces HARMONI's performance significantly in those wavelengths. The figure shows that HARMONI $(\mathrm{R}=4000)$ is very competitive in J and $\mathrm{H}$, especially under LTAO operations (see text for further details).

\section{REFERENCES}

[1] Thatte, N. et al., "HARMONI: a visible and near infrared integral field spectrograph for the E-ELT ", SPIE proc., 7734, (2010).

[2] Fusco, T., Thatte, N., Meimon, S., "Adaptive optics systems for HARMONI: a visible and near-infrared integral field spectrograph for the E-ELT", SPIE proc., 7736, (2010).

[3] Geach, J.E. et al., "HiZELS: a high-redshift survey of $\mathrm{H} \alpha$ emitters - I. The cosmic star formation rate and clustering at $z=2.23 "$, M.N.R.A.S., 388, 1473 (2008).

[4] Sobral, D. et al., "HiZELS: a high-redshift survey of $\mathrm{H} \alpha$ emitters - II. The nature of star-forming galaxies at $\mathrm{z}=$ 0.84", M.N.R.A.S., 398, 75 (2009).

[5] http://www.stsci.edu/jwst/instruments/nirspec/sensitivity/index_html 